\title{
Analysis and Comparison of Optical Performance and Collectible Solar Energy between Multi-sectioned Compound Parabolic Concentrator (CPC) and Restricted Exit Angle CPC
}

\author{
Damasen Ikwaba Paul \\ Department of Physical Sciences, Faculty of Science, Technology and Environmental Studies, The \\ Open University of Tanzania, P. O. Box 23409, Dar es Salaam, Tanzania. \\ E-mail addresses: paul.ikwaba@out.ac.tz; paul.ikwaba2018@gmail.com
}

Received 24 Jun 2020, Revised 9 Mar 2021, Accepted 21 Apr 2021, Published May 2021

DOI: https://dx.doi.org/10.4314/tjs.v47i2.5

\begin{abstract}
This study was conducted to analyse and compare the optical performance and collectible solar radiation energy of two different Compound Parabolic Concentrators (CPCs): multi-sectioned CPC (hereafter called M-CPC) and restricted exit angle CPC (hereafter called R-CPC) so as to ascertain the best CPC for photovoltaics applications. For easy comparison between M-CPC and R-CPC, a standard CPC (hereafter called S-CPC) was also designed. A detailed ray trace simulation analysis was undertaken to compare ray trace diagrams, angular acceptance, optical efficiency and energy flux distribution of the three CPCs. Results indicated that the angular acceptance and optical efficiency of the three CPCs were the same $(100 \%)$ between $0^{\circ}$ and $15^{\circ}$ incidence angles, but significantly varied above $15^{\circ}$. On the other hand, solar radiation distribution on the solar cell was more uniform for the M-CPC than that of S-CPC and R-CPC. In terms of annual solar radiation collection, results indicated that both S-CPC and M-CPC collected approximately the same amount of energy $\left(49,500 \mathrm{~W} / \mathrm{m}^{2}\right)$. Furthermore, the energy collected by SCPC or M-CPC was higher than that collected by R-CPC by about $23 \%$. Therefore, based on the energy flux distribution and collectible solar radiation energy, M-CPC is the best concentrator for photovoltaics applications.
\end{abstract}

Keywords: Multi-sectioned CPC, restricted exit angle CPC, optical performance, collectible solar radiation energy.

\section{Introduction}

The generation of solar electricity from solar energy is the most promising substitute for fossil energy since solar energy is renewable, non-pollutant, free energy source and available everywhere in the world. The importance of solar electricity is clearly seen in off-grid areas where it is used to improve efficiency in sectors such as education, health, agriculture, technology, poverty reduction, gender, clean water and other aspects of human life (IEA 2017). It has also been realised that access to reliable and affordable electricity, in a secure and environmentally friendly manner, is an essential element for achieving the Sustainable Development Goals (SDGs) by 2030 (UN 2014). Despite these facts, solar electricity has not been utilised in those sectors as much as it should have been due to high costs of solar panels. These costs are attributed to the fact that the materials used in making the solar cells and the fabrication processes involved are relatively expensive. Nevertheless, the use of solar concentrators offers a solution to this problem because the optical element of the concentrating system is 
cheaper than solar modules, thus low cost per kilowatt-hour of manufacturing capital. In addition, the use of solar concentrators increases solar energy flux on the solar module which results into significant improvements in the electrical power outputs (Chemisana and Mallick 2013).

Over the past five decades, different types of photovoltaics (PV) concentrating systems have been used for solar electricity generation (Paul 2019a). However, the most popular PV concentrating system is the Compound Parabolic Concentrator, commonly known as CPC (Paul 2019a, b). This collector was invented in $1960 \mathrm{~s}$ but its importance for photovoltaics energy conversion was recognised in the United States in 1975 (Winston 1976). The CPC consists of two distinct parabolic reflectors which direct solar irradiance entering through the aperture to the exit area where the PV module is installed (Figure 1a). The axes of the two parabolic surfaces are oriented away from the axis of the $\mathrm{CPC}$ by an angle called acceptance half-angle ( $\theta_{a}$ ) which is the angular range over which all rays incident on the aperture are accepted without moving all or part of the CPC. Winston (1974) proved that a CPC is an ideal collector because any ray (direct or diffuse) incident at the aperture within the acceptance angle will reach the PV module whilst all others will be reflected back and re-emerge back through the entry aperture.

Due to variations in geographical locations and energy requirements, CPC is classified as symmetric or asymmetric (Rabl 1976a). Both symmetric and asymmetric CPCs have similar advantages over other PV concentrators which include high optical efficiency (Rabl 1976a, b), minimum errors of alignment (O'Gallagher and Winston 1983, Abdullahi et al. 2013), ability to be used as static collector (O'Gallagher and Winston 1983) and being able to collect direct irradiance as well as diffuse irradiance (Rabl 1976a, b). However, one of the fundamental drawbacks of CPCs to PV application is that they distribute solar radiation intensity on the surface of the PV module non-uniformly (Sangrador and Sala 1979). When the solar cells in the PV module or array are nonuniformly illuminated, the solar cells that receive less energy flux generate less current than the fully illuminated solar cells. Yet, the least-illuminated solar cells are also forced to carry the same high current as the other fully illuminated solar cells. As a result, the leastilluminated solar cells operate under reverse bias, thus dissipating power in the form of heat (hotspots) (Pfeiffer and Bihler 1982). For a solar panel in which all solar cells are connected in series, such hotspots cause reduction in power output due to the fact that the current of the entire solar panel depends on the least-illuminated solar cell(s) (James and Williams 1978, Edenburn and Burns 1981, Paul et al. 2013). Therefore, high radiation intensity on the PV module is lost since the intensity above the least-illuminated cells does not contribute to power generation.

To attain uniform energy flux distribution on the surface of the PV module with the CPC, researchers have suggested different modifications on the reflecting surfaces of a standard CPC shown in Figure 1a (Paul 2019a). For example, Paul (2019c) proposed a discrete $\mathrm{CPC}$ in which its reflector surfaces consist of a series of flat reflector sections of different widths that are oriented at different angles (Figure 1b). On the other hand, Yu et al. (2014) proposed a CPC called restricted exit angle (Figure 1c) in which its reflector surfaces consist of two parts: the upper part which is a parabolic section and the lower part which is a straight section. Simulation results revealed that energy flux distribution on the solar cell for both CPCs (discrete CPC and restricted exit angle CPC) was more uniform than similar standard CPC (Yu et al. 2014, Paul 2019c). However, the power output generated by any PV-CPC system depends on many factors, including uniformity of energy flux distribution on the surface of the solar cell, optical efficiency and solar energy collected by the CPC. Therefore, the aim of this work was to analyse and compare the optical performance 
and collectible solar radiation energy of two different CPCs (multi-sectioned CPC and restricted exit angle $\mathrm{CPC}$ ) so as to ascertain the

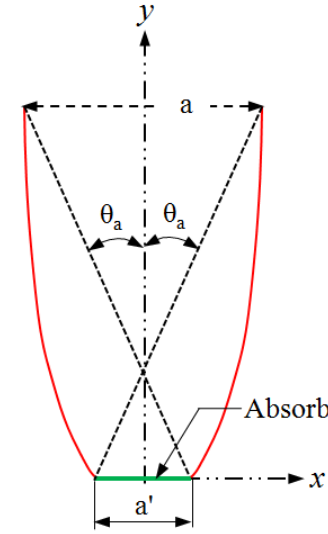

(a)

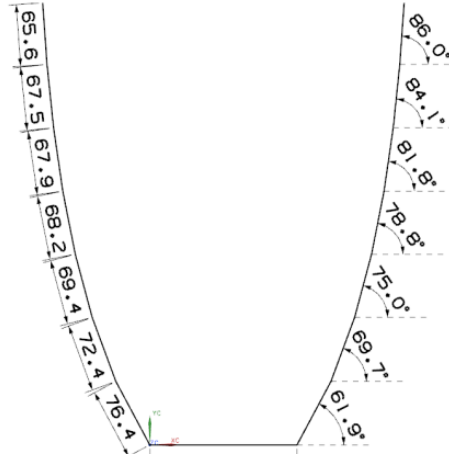

(b) best CPC design for solar electricity generation.

Figure 1: Cross-sectional geometries of CPC reflector surfaces (a) Standard CPC (Rabl 1976a) (b) Multi-sectioned CPC [all measurements related to lengths are in mm] (Paul 2019c) (c) Restricted exit angle CPC (Yu et al. 2014).

\section{Materials and Methods}

\section{Design of the CPCs}

In this work, three different CPCs, namely standard symmetric CPC, symmetric multisectioned CPC and symmetric restricted exit angle CPC were designed. Standard CPC was used as a reference collector for easy comparison between multi-sectioned CPC and restricted exit angle CPC. The detail designs of the three CPCs are presented in the subsections below.

\section{Design of standard CPC}

Figure 1(a) shows the cross-sectional geometries of the standard CPC where $a$ and $a$ ' are the aperture area and exit area of the CPC, respectively, while $\theta_{\mathrm{a}}$ is the acceptance halfangle. The design of such CPC is based on the

$$
\left.\begin{array}{l}
f_{R}=\left(1+\sin \left(\theta_{a R}\right)\right) \times \frac{w_{r}}{2} \\
f_{L}=\left(1+\sin \left(\theta_{a L}\right)\right) \times \frac{w_{r}}{2}
\end{array}\right\}
$$

edge-ray principle which requires that all rays incident upon the entry aperture (at the limiting acceptance angle) must be reflected directly to the edge of the exit aperture (Welford and Winston 1978). Based on this principle, the reflector profiles of symmetric CPCs can be designed using different equations (Paul 2019b). However, in this study the co-ordinates for the S-CPC reflector surfaces were generated using Equation (1) (Paul 2019b, c). Table 1 shows the geometrical properties of $S$ CPC before and after truncation. In Table 1, the acceptance half-angle of S-CPC was chosen to be $23.5^{\circ}$ in order to accept a large proportion of diffuse radiation incident on the aperture and concentrate it without the need of a tracking device (Sørensen et al. 2009). 


$$
\begin{aligned}
& \left(x_{R}, y_{R}\right)=\left(\frac{2 f_{R} \sin \theta}{1+\cos \theta}, \frac{-2 f_{R} \cos \theta}{1+\cos \theta}\right) \quad \text { for } \frac{\pi}{2}-\theta_{a R} \leq \theta \leq \pi-\theta_{a R}-\theta_{a L} \\
& \left.\left(x_{L}, y_{L}\right)=\left(\frac{2 f_{L} \sin \theta}{1+\cos \theta}, \frac{-2 f_{L} \cos \theta}{1+\cos \theta}\right) \quad \text { for } \frac{\pi}{2}-\theta_{a L} \leq \theta \leq \pi-\theta_{a R}-\theta_{a L}\right) \\
& \left(x_{R}^{\prime}, y_{R}^{\prime}\right)=\left\lfloor\left(x_{R} \cos \left(-\theta_{a R}\right)+y_{R} \sin \left(-\theta_{a R}\right)\right),\left(-x_{R} \sin \left(-\theta_{a R}\right)+y_{R} \cos \left(-\theta_{a R}\right)\right)\right\rfloor \\
& \left.\left(x^{\prime}{ }^{,} y_{L}^{\prime}\right)=\left[\left(x_{L} \cos \left(-\theta_{a L}\right)+y_{L} \sin \left(-\theta_{a L}\right)\right),\left(-x_{L} \sin \left(-\theta_{a L}\right)+y_{L} \cos \left(-\theta_{a L}\right)\right)\right]\right\} \\
& \left.\begin{array}{l}
x_{R-\text { mirror }}=-x_{L}^{\prime} \\
y_{L-\text { mirror }}=y_{L}^{\prime}
\end{array}\right\} \\
& \left.\begin{array}{l}
x_{R-\text { mirror }}=x_{L-\text { mirror }}+w_{r} \\
y_{L-\text { mirror }}=y_{L-\text { mirror }}
\end{array}\right\}
\end{aligned}
$$

where:

- $\left(f_{R}, f_{L}\right)$ is right and left focal distances [which are used to calculate the $(x, y)$ co-ordinates of the profiles],

- $w_{r}$ is the width of the PV module,

- $\theta_{a R}$ and $\theta_{a L}$ are acceptance halfangles for the right and left parabolas, respectively. For symmetric $2 \mathrm{D} \mathrm{CPC}$, $\theta_{a R}=\theta_{a L}$,
- $\quad x$ and $y$ are the co-ordinates for each point on the right $\left(x_{R}, y_{R}\right)$ and left $\left(x_{L}, y_{L}\right)$ parabolas,

- $\quad\left(x^{\prime}, y^{\prime}\right)$ are the $x$ and $y$ co-ordinates of the new parabola profiles,

- $x_{R-\text { mirror }}$ and $y_{L-m i r r o r}$ are the image co-ordinates for each point on the right and left parabola.

Table 1: Values of various parameters of S-CPC

\begin{tabular}{lll}
\hline Parameter & Before truncation & After truncation \\
\hline Acceptance half-angle, $\theta_{a}\left(^{\circ}\right)$ & 23.50 & 24.04 \\
Concentration ratio, $\mathrm{C}_{\mathrm{g}}[-]$ & 2.51 & 2.46 \\
Aperture width, $\mathrm{A}_{\mathrm{a}}(\mathrm{mm})$ & 391.4 & 383.0 \\
PV module width, $w_{\mathrm{r}}(\mathrm{mm})$ & 156 & 156 \\
PV module length $(\mathrm{mm})$ & 156 & 156 \\
Maximum height, $\mathrm{H}_{\max }(\mathrm{mm})$ & 627.1 & 469.1 \\
Collector maximum length $(\mathrm{mm})$ & 1,000 & 1,000 \\
\hline
\end{tabular}

\section{Design of multi-sectioned CPC}

In this study, a multi-sectioned symmetric CPC consisting of seven flat reflector sections of different widths and oriented at different angles was designed (Figure 1b). For direct comparison of the results, M-CPC was deliberately designed to have the same geometrical values as S-CPC after truncation as shown in Table 2. The design of this collector was based on Equation (2) (Paul 2019b). 


$$
\begin{gathered}
w_{1}=\frac{H_{\max }}{N_{T}} \pm y \\
w_{p}=\left(\frac{H_{\max }}{N_{T}} \times p\right) \pm y=\left(w_{1} \times p\right) \pm y
\end{gathered}
$$

where:

- $w_{1}$ is the width of the first flat reflector section (counted from the exit aperture),

- $N_{T}$ is the total number of flat reflector sections in the collector,

- $y$ is the distance to the nearest $y$-coordinate of S-CPC,

- $\quad p$ is the position of the flat reflector section in the concentrator (counted from the exit aperture towards the entry aperture),

- $w_{p}$ is the width of the $p$ flat reflector sections in the concentrator.

Table 2: Values of various parameters of $\mathrm{M}$ CPC

\begin{tabular}{ll}
\hline Parameter & Value \\
\hline Acceptance half-angle, $\theta_{a}\left(^{\circ}\right)$ & 24.04 \\
Concentration ratio, $\mathrm{C}_{\mathrm{g}}[-]$ & 2.46 \\
Aperture width, $\mathrm{A}_{\mathrm{a}}(\mathrm{mm})$ & 383 \\
PV module width, $w_{\mathrm{r}}(\mathrm{mm})$ & 156 \\
PV module length $(\mathrm{mm})$ & 156 \\
Maximum height, $\mathrm{H}_{\max }(\mathrm{mm})$ & 469.1 \\
Total number of flat reflector & 7 \\
sections, $N_{T}$ [-] & \\
Collector maximum length $(\mathrm{mm})$ & 1,000 \\
\hline
\end{tabular}

\section{Design of restricted exit angle $\mathrm{CPC}$}

Figure 1(c) shows the cross-sectional geometries of a restricted exit angle CPC for this study. To design such a collector, Equations (3a) and (3b) were used to generate the co-ordinates for the right-hand side (RHS) parabola and straight line section, respectively (Yu et al. 2014). The left-hand side (LHS) of the R-CPC was obtained from the mirror image of the RHS, i.e., $y(R H S)=y(L H S)$ and
$x(R H S)=-x(L H S) \quad($ Gordon $\quad 1986$,

O'Gallagher 2008).

$$
\begin{aligned}
& x=\frac{a^{\prime}\left(\sin \theta_{\mathrm{e}}+\sin \theta_{\mathrm{a}}\right) \sin \theta}{1-\cos \left(\theta+\theta_{\mathrm{a}}\right)}-0.5 a^{\prime} \\
& y=\frac{a^{\prime}\left(\sin \theta_{\mathrm{e}}+\sin \theta_{\mathrm{a}}\right) \sin \theta}{1-\cos \left(\theta+\theta_{\mathrm{a}}\right)} \\
& \text { for } \theta_{\mathrm{a}} \leq \theta \leq \theta_{\mathrm{e}} \\
& y=\tan \beta_{\mathrm{st}}\left(x-0.5 a^{\prime}\right) \text { for } \\
& 0.5 a^{\prime} \leq x \leq r_{0} \sin \theta_{\mathrm{e}}-0.5 a^{\prime}
\end{aligned}
$$

where: $a^{\prime}$ is the half-width of the absorber, $\theta$ is the polar angle of point $P$ on the parabola as shown in Figure 1(c) and is measured from the $y$-axis, $\theta_{\mathrm{a}}$ is the acceptance half-angle, $\theta_{\mathrm{e}}$ is the maximum exit angle of the CPC for the radiation within its acceptance angle, $\beta_{\text {st }}$ is the tilt angle of the straight section in Figure 1(c) relative to the absorber ( $x$-axis) which is equal to the tilt angle of the tangent line passing through $\mathrm{D}$ (the lower end of the parabolic section) and $r_{0}$ is the length of line $\mathrm{B}^{\prime} \mathrm{D}$ in Figure 1(c). Angle $\beta_{\text {st }}$ and the value of $r_{0}$ were calculated from Equations (3c) and (3d), respectively (Yu et al. 2014).

$$
\begin{aligned}
& \beta_{\mathrm{st}}=0.5 \pi-0.5\left(\theta_{\mathrm{e}}-\theta_{\mathrm{a}}\right) \\
& r_{0}=\frac{a^{\prime}\left(\sin \theta_{\mathrm{a}}+\sin \theta_{\mathrm{e}}\right)}{1-\cos \left(\theta_{\mathrm{a}}+\theta_{\mathrm{e}}\right)}
\end{aligned}
$$

For the purpose of having the same concentration ratio as that of S-CPC and MCPC, R-CPC was designed based on the values of the parameters listed in Table 3. Figure 2 shows the 3D view of S-CPC, M-CPC and R$\mathrm{CPC}$ having the same concentration ratio of 2.46 . 
Paul - Analysis and comparison of optical performance and collectible solar energy ...

Table 3: Values of various parameters of R-CPC having the same concentration ratio as S-CPC and M-CPC

\begin{tabular}{lc}
\hline Name of the parameter & Value \\
\hline Acceptance half-angle, $\theta_{a}\left({ }^{\circ}\right)$ & 21.20 \\
Concentration ratio, $\mathrm{C}_{\mathrm{g}}[-]$ & 2.46 \\
Aperture width, $\mathrm{A}_{\mathrm{a}}(\mathrm{mm})$ & 383.99 \\
PV module width, $w_{\mathrm{r}}(\mathrm{mm})$ & 156 \\
PV module length $(\mathrm{mm})$ & 156 \\
Maximum height, $\mathrm{H}_{\max }(\mathrm{mm})$ & 543.6 \\
Collector maximum length $(\mathrm{mm})$ & 1,000 \\
\hline
\end{tabular}

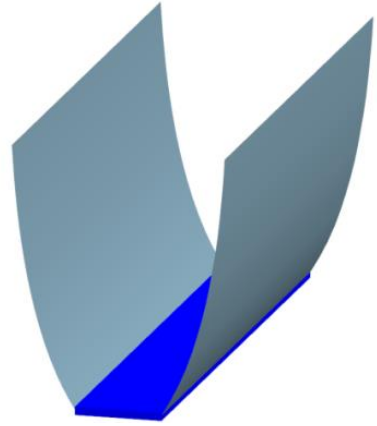

(a)

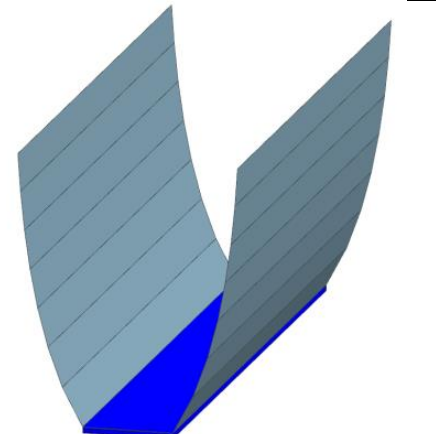

(b)

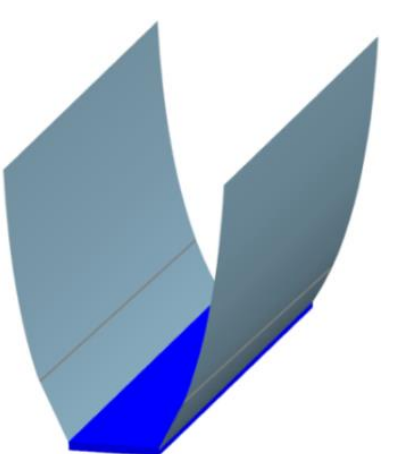

(c)

Figure 2: Geometries of the CPCs in 3D view (a) S-CPC (b) M-CPC (c) R-CPC (all CPCs have the same concentration ratio of 2.46).

\section{Optical performance analysis}

The optical performance of each CPC was analysed using ray tracing simulation program (Zacharopoulos 2001) which is based on the laws of reflection and refraction of light (Welford and Winston 1978). For each CPC, the optical performance was analysed in terms of ray trace diagrams, angular acceptance, optical efficiency and energy flux distribution along the solar cell.

$$
E(t)=A_{S C} C_{\mathrm{g}} \alpha_{S C}\left\{\left(G_{B, C P C} \times \eta_{B, \text { optical }}\right)+\left(G_{D, C P C} \times \eta_{D, \text { optical }}\right)\right\}
$$

where: $G_{B, C P C}$ is the beam solar irradiance on the aperture of the CPC, $G_{D, C P C}$ is the diffuse solar irradiance on the aperture of the CPC, $C_{g}$ is the geometrical concentration ratio of the CPC, $\eta_{B \text {,optical }}$ is the optical efficiency of the CPC for beam component of solar irradiance, $\eta_{D \text {,optical }}$ is the optical efficiency of the CPC for diffuse component of solar irradiance, $A_{S C}$ is the active area of the solar 
cell placed underneath of the CPC, and $\alpha_{S C}$ is the absorptance of the solar cell.

The beam solar irradiance on the aperture of the CPC in Equation (4) was calculated from Equation (5a) (Duffie and Beckman 2013).

$$
G_{B, C P C}=F G_{B N} \cos \theta
$$

where: $G_{B N}$ is the average hourly beam normal irradiance, $F$ is the control function

$$
\begin{aligned}
& \left(\beta-\theta_{a}\right) \leq \tan ^{-1}\left(\tan \theta_{z} \times \cos \gamma_{S}\right) \leq\left(\beta+\theta_{a}\right) \\
& \cos \theta=\cos (\phi-\beta) \cos \delta \cos \omega+\sin (\phi-\beta) \sin \delta
\end{aligned}
$$

where: $\phi$ is the latitude of the location, $\beta$ is the tilt angle of the CPC ( $\beta=\phi$ for Dar es Salaam, Tanzania ), $\theta_{a}$ is acceptance half-angle of the CPC, $\omega$ is the hour angle which is the angular displacement of the sun east or west of the local meridian due to rotation of the earth on its axis at $15^{\circ}$ per hour (morning negative and afternoon positive), $\theta_{z}$ is the zenith angle calculated from Equation (5d), $\gamma_{S}$ is the solar azimuth angle given by Equation (5e) and $\delta$ is the declination angle calculated from Equation (5f) (Duffie and Beckman 2013).

$$
G_{D, C P C}= \begin{cases}\frac{G_{D}}{C_{g}} & \text { if }\left(\beta+\theta_{a}\right)<90^{\circ} \\ \frac{G_{D}}{2}\left(\frac{1}{C_{g}}+\cos \beta\right) & \text { if }\left(\beta+\theta_{a}\right)>90^{\circ}\end{cases}
$$

where: $G_{D}$ is the average hourly diffuse horizontal solar irradiance, obtained from SoDa website (SoDa 2019).

In this study, $C_{g}=2.46$ as indicated in Tables 1, 2 and 3, $\alpha_{S C}=1$ because solar cells are considered to be perfect absorbers at any incidence angle (Green 2006), $A_{S C}=0.0243 \mathrm{~m}^{2}$ (from Tables 1, 2 and 3), $\eta_{B, \text { optical }}$ was obtained from ray-tracing simulation as pointed out under optical $\cos \theta_{z}=\cos \phi \cos \delta \cos \omega+\sin \phi \sin \delta \quad(5 \mathrm{~d})$

$$
\begin{aligned}
& \gamma_{S}=\operatorname{sign}(\omega)\left|\cos ^{-1}\left(\frac{\cos \theta_{z} \sin \phi-\sin \delta}{\sin \theta_{z} \cos \phi}\right)\right| \\
& \delta=23.45^{\circ} \sin \left(360 \frac{284+n}{365}\right)
\end{aligned}
$$

where: $n$ is the day number counted from the beginning of the year, with January $1^{\text {st }}$ as $n=1$.

The values of $G_{D, C P C}$ in Equation (4) for each day were calculated using Equation (6a) (Duffie and Beckman 2013).

performance analysis section and $\eta_{D \text {,optical }}$ was estimated as recommended by $\mathrm{Su}$ et al. (2012).

\section{Results and Discussions \\ Optical performance}

\section{Ray trace diagrams}

The interactions of sunlight with PV-CPC for each collector (S-CPC, M-CPC and R-CPC) at different incidence angles $\left(0^{\circ}, 10^{\circ}\right.$ and $\left.20^{\circ}\right)$ are shown in Figure 3. It can be seen that the amount of solar radiation energy collected by the PV cell is a function of both CPC reflector 
surface geometry and incidence angle. Both SCPC and R-CPC accept all sunlight incidents on the aperture area at each incidence angle. However, as the sunlight incident angle increases from the perpendicular of the aperture, the effect of CPC reflector surface geometry is clearly observed. For example, MCPC (Figure 3f) rejects some incident sunlight (shown by blue colour) when the incidence angle is greater than the acceptance angle of the collector after sunlight reflections. In practice, this signifies that M-CPC will generate less electrical energy compared to other CPCs (Figures $3 \mathrm{c}$ and 3i) early in the morning and late hours of the day (with the assumption that the effects of other parameters such as cell temperature, series resistance, energy non-uniformity are kept constant).

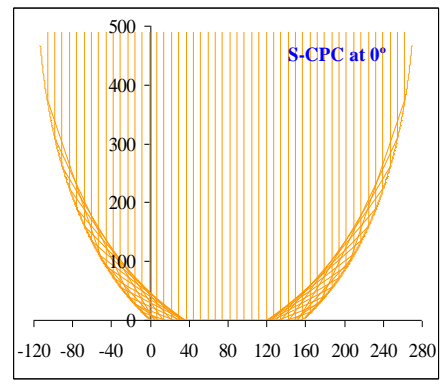

(a)

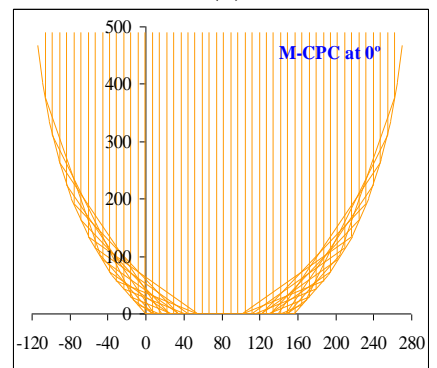

(d)

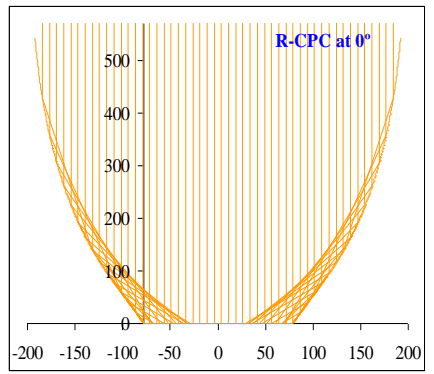

(g)

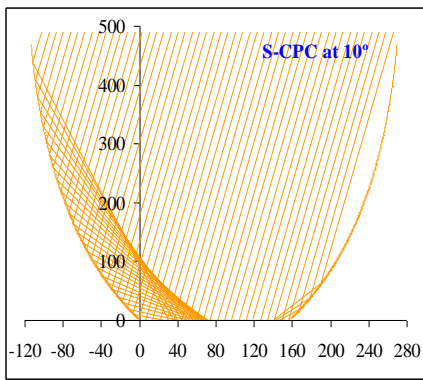

(b)

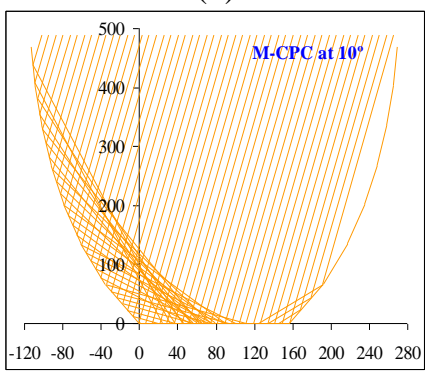

(e)

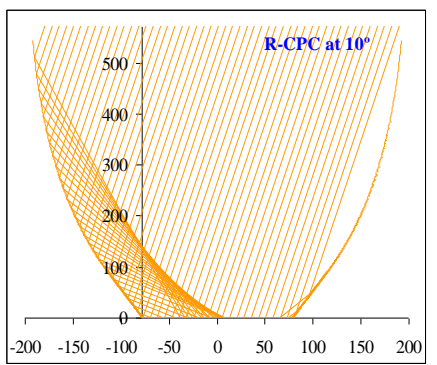

(h)

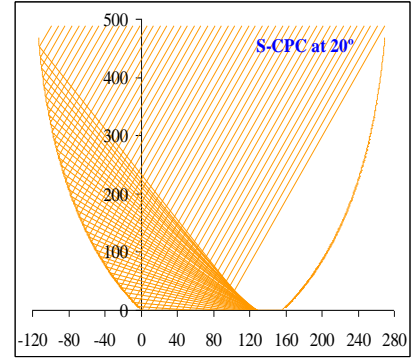

(c)

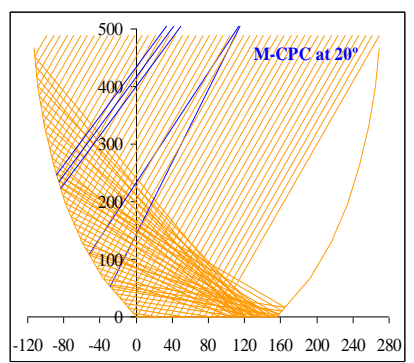

(f)

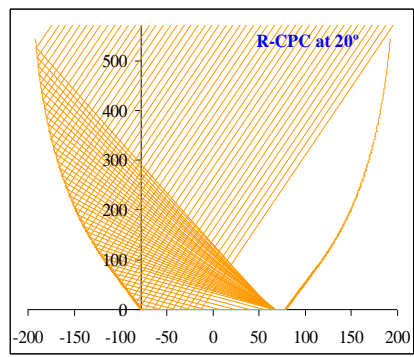

(i)

Figure 3: Variations in sunlight interaction with PV-CPC as a function of collector reflector surface geometry and incidence angle.

\section{Angular acceptance}

The comparison of angular acceptance of the three CPCs at different incidence angles is given in Figure 4. It can be seen that all the light rays incident at the aperture area of each $\mathrm{CPC}$ reach the $\mathrm{PV}$ cell for all the incidence 
angles between $0^{\circ}$ and $15^{\circ}$ because they are within the acceptance angle limits. However, due to differences in reflector surface geometry of each CPC, the angular acceptance varies above $15^{\circ}$. For example, while S-CPC and RCPC attain $100 \%$ at $20^{\circ}, \mathrm{M}-\mathrm{CPC}$ accepts about $95 \%$ only at the same angle. This is due to sunrays that are being rejected by the collector as illustrated in Figure 3f. The rejected rays are due to incident angle being greater than the acceptance angle of the collector after sunlight reflections. It should also be noted that even though the CPCs have the same concentration ratios and aperture areas (as shown in Tables 1, 2 and 3), each CPC has dissimilar angular acceptance characteristic beyond its acceptance angle limits due to variation in reflector surface geometry and maximum height. For example, above $24^{\circ}$, M-CPC has higher angular acceptance than both S-CPC and R-CPC.

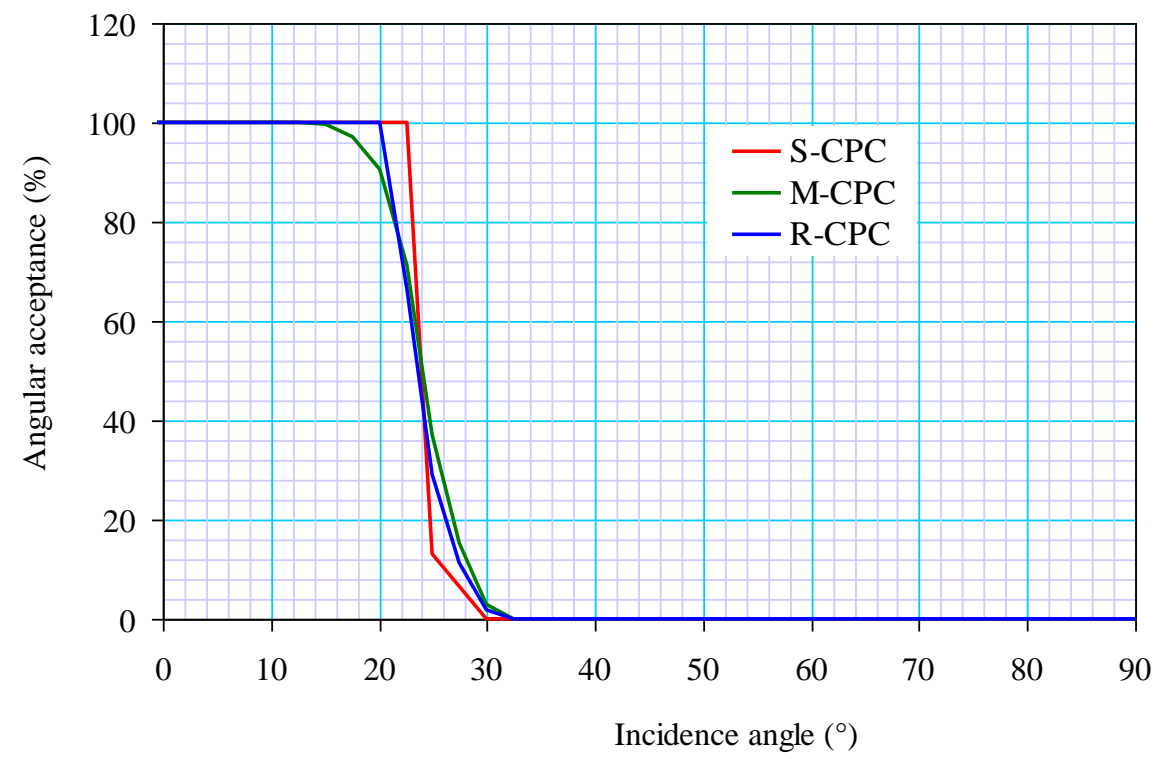

Figure 4: Variation in angular acceptance as the function of CPC reflecting surface geometry and incidence angle.

\section{Optical efficiency}

Figure 5 shows the comparison of the optical efficiencies of the three CPCs at different solar incidence angles. It can be seen that all the collectors have high and similar optical efficiencies in a wide range of solar incidence angles $\left(-15^{\circ}\right.$ and $\left.+15^{\circ}\right)$. This is due to the fact that all light rays are accepted by each collector as illustrated in Figure 4. In addition, most of the incident light rays reach the PV cell either directly or after one reflection as shown in Table 4, which results in very low optical loss at each incidence angle and hence high optical efficiency. However, above $\pm 15^{\circ}$ and up to $\pm 22.5^{\circ}$, the optical efficiency of M-CPC is less that of S-CPC and R-CPC due to variation in reflector surface geometries which gives rise to rejection of some light of rays (Figure $3 \mathrm{f}$ ). Therefore, the optical efficiency of R-CPC is exactly the same as that of S-CPC (for all incidence angles) but for M-CPC, the optical efficiency is less than that of S-CPC in the range of $\pm 15^{\circ}$ to $\pm 22.5^{\circ}$. 
Paul - Analysis and comparison of optical performance and collectible solar energy ...

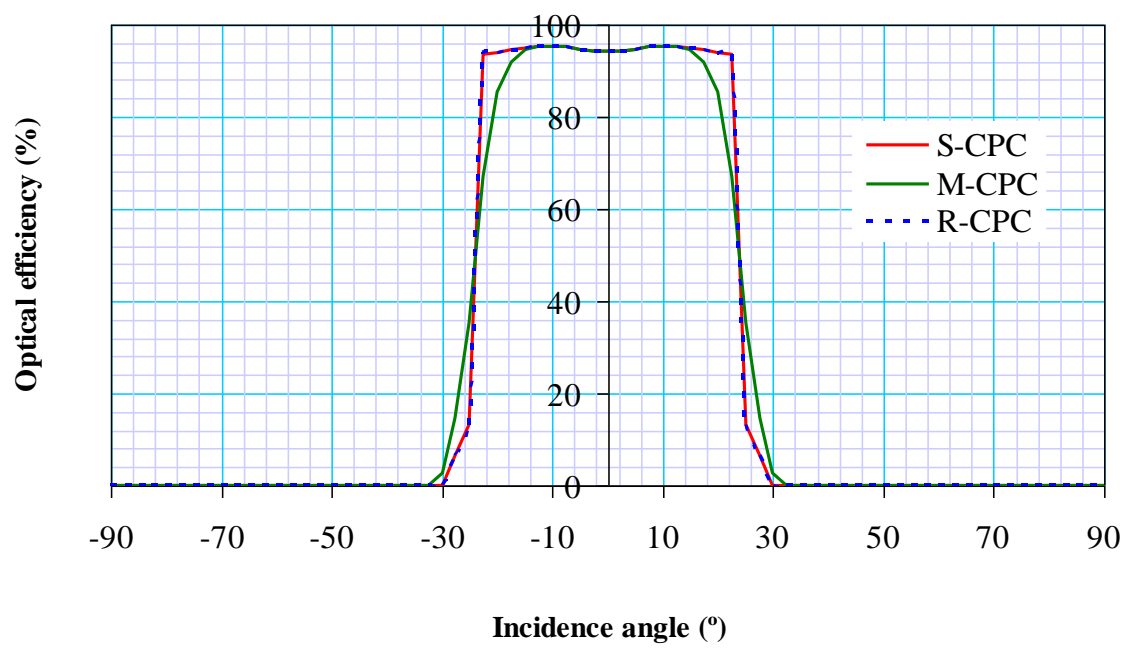

Figure 5: Variation in optical efficiency as a function of CPC reflecting surface geometry and solar incidence angle.

Table 4: Variation of light rays reaching the PV cell directly, after one or multiple reflections as a function of CPC reflector surface geometry and solar incidence angle

Number of light rays (\%) reaching the PV cell directly (zero reflection), after one reflection or multiple reflections at different incidence angles

\begin{tabular}{|c|c|c|c|c|}
\hline \multirow{2}{*}{ Incidence angle } & \multirow{2}{*}{$\begin{array}{l}\text { Number of } \\
\text { reflections }\end{array}$} & \multicolumn{3}{|c|}{ Type of CPCs } \\
\hline & & S-CPC & $\mathrm{M}-\mathrm{CPC}$ & R-CPC \\
\hline \multirow[t]{4}{*}{$\mathbf{0}^{\mathbf{0}}$} & 0 & 40 & 40 & 40 \\
\hline & 1 & 52 & 52 & 52 \\
\hline & 2 & 8 & 8 & 8 \\
\hline & 3 & 0 & 0 & 0 \\
\hline \multirow[t]{4}{*}{$5^{\circ}$} & 0 & 52 & 42 & 42 \\
\hline & 1 & 54 & 54 & 54 \\
\hline & 2 & 4 & 4 & 4 \\
\hline & 3 & 0 & 0 & 0 \\
\hline \multirow[t]{4}{*}{$10^{\circ}$} & 0 & 40 & 40 & 42 \\
\hline & 1 & 60 & 60 & 58 \\
\hline & 2 & 0 & 0 & 0 \\
\hline & 3 & 0 & 0 & 0 \\
\hline \multirow[t]{4}{*}{$15^{\circ}$} & 0 & 38 & 38 & 32 \\
\hline & 1 & 62 & 62 & 68 \\
\hline & 2 & 0 & 0 & 0 \\
\hline & 3 & 0 & 0 & 0 \\
\hline \multirow[t]{4}{*}{$20^{\circ}$} & 0 & 26 & 26 & 18 \\
\hline & 1 & 74 & 64 & 82 \\
\hline & 2 & 0 & 0 & 0 \\
\hline & 3 & 0 & 0 & 0 \\
\hline
\end{tabular}




\section{Energy flux distribution along the solar cell} Figure 6 shows the comparison of solar energy concentration across the solar cell for the three CPCs for the selected incidence angles $\left(0^{\circ}, 5^{\circ}\right.$, $10^{\circ}, 15^{\circ}$ and $20^{\circ}$ ). It is evident from these figures that the distribution of solar energy for each CPC is non-uniform and the magnitude of uniformity varies with the reflector surface geometry of the CPC and the incidence angle. However, at each incidence angle, the energy flux concentration across the solar cell for $\mathrm{M}$ CPC is more uniform than that of S-CPC and R-CPCs as illustrated in Figure 6 and Table 5. In practical situation this implies that, at each incident angle, the solar panel with M-CPC will generate more electrical power than that with S-CPC or R-CPC. On the other hand, R$\mathrm{CPC}$ will generate more electrical power than $\mathrm{S}-\mathrm{CPC}$ only between $0^{\circ}$ to $10^{\circ}$ incidence angles because the energy concentration distribution is less than that of S-CPC. Nevertheless, above $10^{\circ}, \mathrm{R}-\mathrm{CPC}$ has no advantage over the standard CPC because of high non-uniform energy concentration. Therefore, if solar radiation uniformity across the surface of the solar panel is taken as the only criterion for choosing the best CPC for solar power generation, then it is advantageous to use M-CPC than S-CPC or RCPC.

Table 5: Variation of maximum solar energy concentration across the solar cell as function of CPC reflector surface geometry and solar radiation incidence angle

\begin{tabular}{cccc}
\hline Incidence & \multicolumn{3}{l}{ Maximum solar energy concentration for each collector } \\
\cline { 2 - 4 } angle & S-CPC & M-CPC & R-CPC \\
\hline $0^{\circ}$ & 5.06 & 1.69 & 2.47 \\
$5^{\circ}$ & 7.30 & 1.78 & 3.78 \\
$10^{\circ}$ & 8.96 & 1.91 & 8.51 \\
$15^{\circ}$ & 12.26 & 2.39 & 11.59 \\
$20^{\circ}$ & 18.11 & 1.89 & 30.30 \\
\hline
\end{tabular}

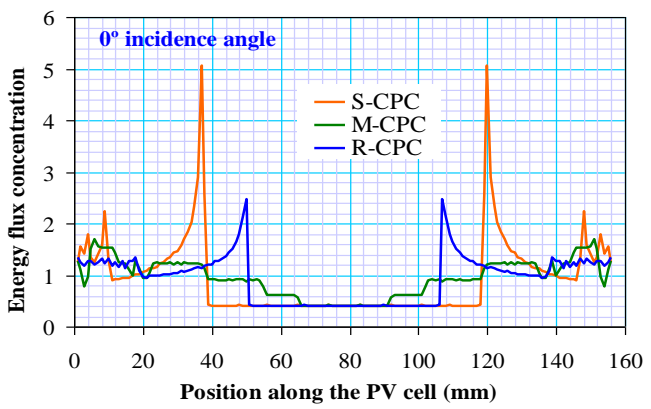

(a)

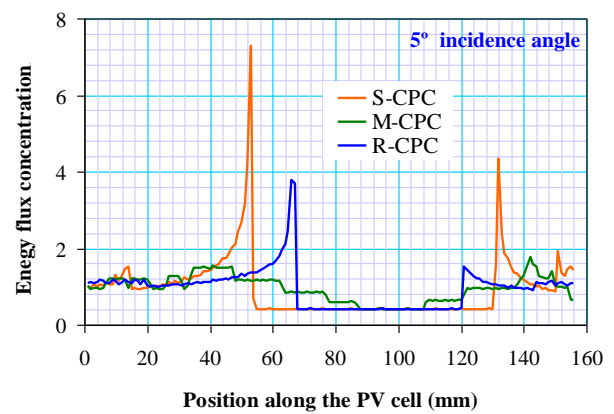

(b) 


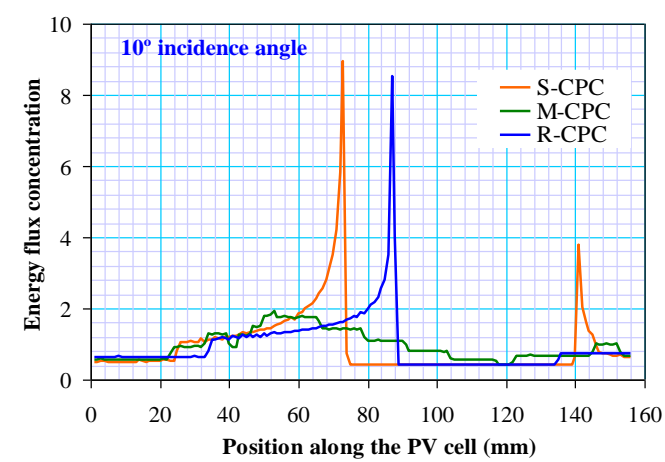

(c)

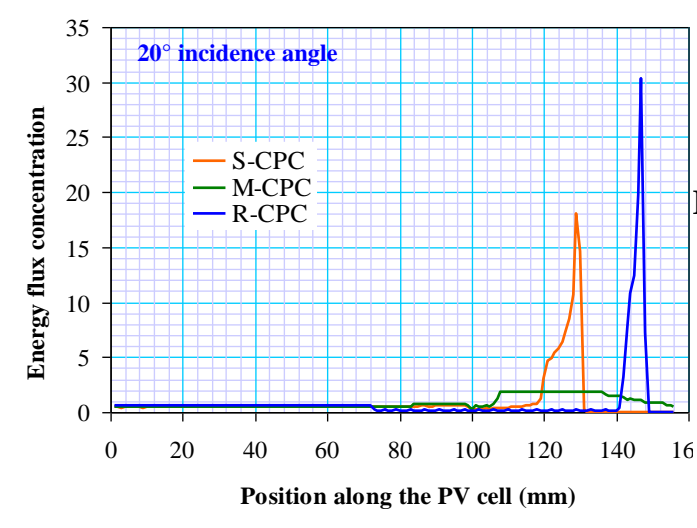

\section{Solar radiation collection}

The effect of CPC reflector surface geometry on solar radiation collection was investigated and the results are presented in Figure 7. It can be seen that the amount of solar radiation collected by S-CPC and M-CPC in each month was always the same throughout the year. This is attributed to both CPCs having the same acceptance half-angle and approximately similar optical efficiencies. However, solar radiation collected by $\mathrm{S}-\mathrm{CPC}$ or $\mathrm{M}-\mathrm{CPC}$ in January, February, May, June, July, August, October, November and December was higher than the energy collected by R-CPC by about $87 \%, 4 \%, 42 \%, 413 \%, 77 \%, 6 \%, 3 \%, 66 \%$ and $153 \%$, respectively. This is due to the fact that R-CPC has different reflector geometry compared to S-CPC or M-CPC; hence less

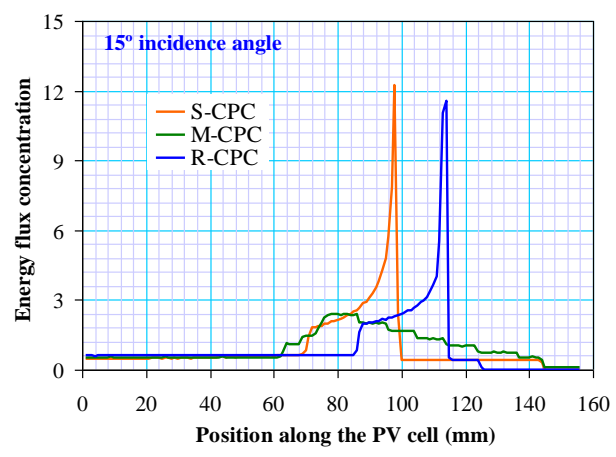

(d)

Figure 6: Variation in solar energy distribution along the PV cell at different incidence angles for the three CPCs.

solar radiation collection hours per day. On the other hand, when the comparison of the three CPCs was made based on the annual solar radiation collection (Figure 8), it was found that both S-CPC and M-CPC collected approximately the same amount of energy $\left(49,500 \mathrm{~W} / \mathrm{m}^{2}\right)$. Besides, the energy collected by S-CPC or M-CPC was higher than that collected by R-CPC by about $23 \%$. This implies that over the whole year, R-CPC will generate lower power output compared to SCPC or M-CPC (keeping other factors constant). This analysis and the results of energy flux distribution along the solar cell presented in Figure 6 leads to the conclusion that $\mathrm{M}-\mathrm{CPC}$ is the best collector for PV applications. 


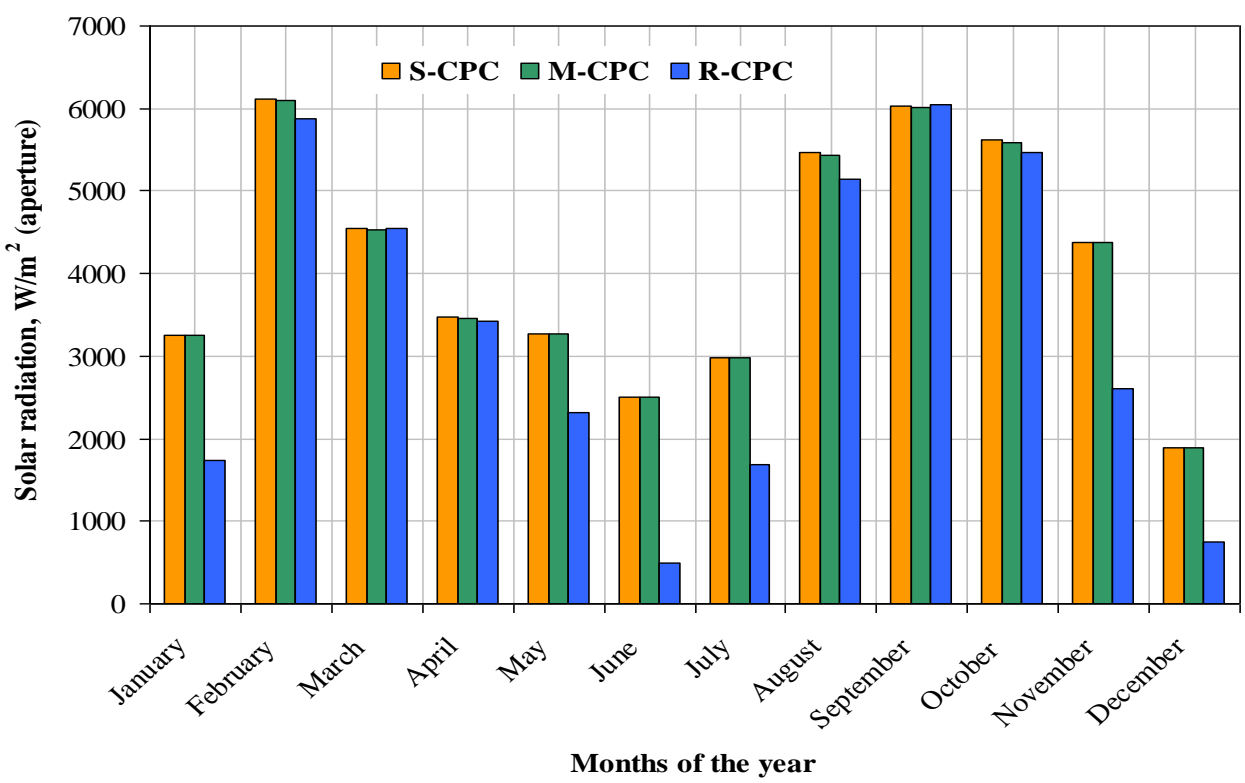

Figure 7: Comparison of solar radiation (direct and diffuse) collected by each CPC in different months.

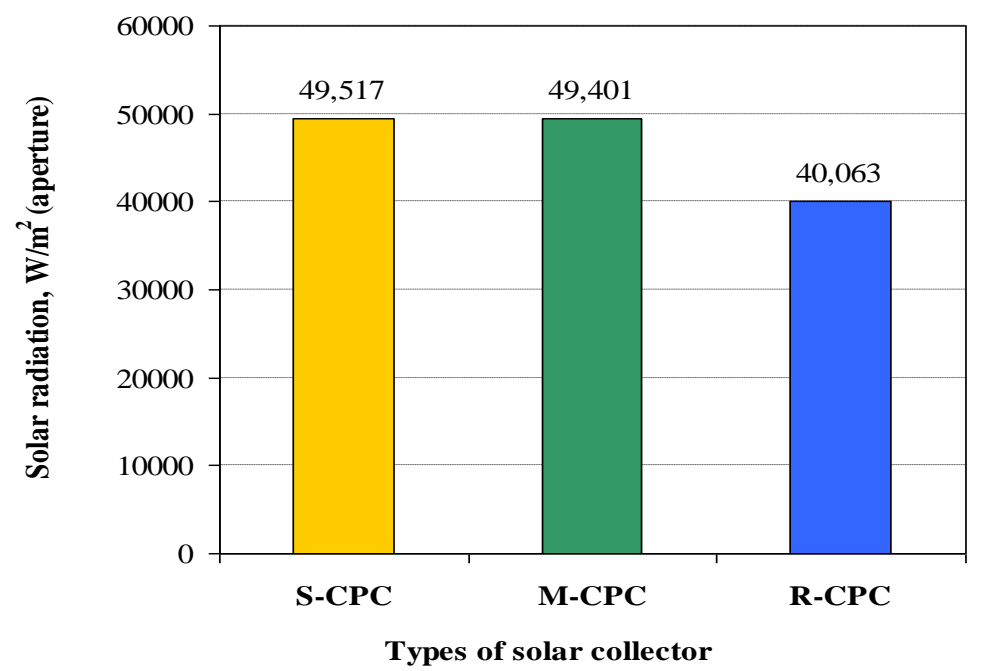

Figure 8: Annual solar energy collected by different CPCs.

\section{Conclusion}

This study found that the angular acceptance and optical efficiency of the three CPCs were the same $(100 \%)$ between $0^{\circ}$ and $15^{\circ}$ incidence angles but significantly varied above $15^{\circ}$. For example, while S-CPC and R-CPC attained $100 \%$ at $20^{\circ}$, M-CPC accepted only about $95 \%$ at the same angle. Furthermore, above $\pm 15^{\circ}$ and up to $\pm 22.5^{\circ}$, the optical efficiency of M$\mathrm{CPC}$ was less that of S-CPC and R-CPC due to 
variation in reflector surface geometry which gives rise to rejection of some light of rays. In contrast, solar radiation intensity on the solar cell was more uniform for M-CPC than that of S-CPC and R-CPC. In terms of annual solar radiation accumulation, results indicated that both S-CPC and M-CPC collected approximately the same amount of energy $\left(49,500 \mathrm{~W} / \mathrm{m}^{2}\right)$. Besides, annual solar radiation energy collected by S-CPC or M-CPC was higher than that collected by R-CPC by about $23 \%$. Therefore, based on the solar radiation intensity distribution on the surface of the solar cell and collectible solar radiation energy, MCPC was found to be the best concentrator for photovoltaics applications.

\section{Acknowledgement}

The author thanks Chosun University (Republic of Korea) for their financial support. Indeed, without this funding, this study would have not been conducted.

\section{Conflicts of Interest}

The author declares that there is no conflict of interest regarding this article.

\section{References}

Abdullahi B, AL-Dadah RK and Mahmoud S 2013 Effect of acceptance angle on the design and performance of a heat pipe based compound parabolic collector at Kano, Nigeria. Renew. Energy Power Quality J. 1(11): 1238-1243.

Chemisana D and Mallick TK 2013 Building integrated concentrating solar systems. In: Enteria N and Akbarzadeh A (Eds) Solar Energy Sciences and Engineering Applications $1^{\text {st }}$ ed. (pp. 508-552), Taylor and Francis Group, London.

Duffie JA and Beckman WA 2013 Solar Engineering of Thermal Processes. $4^{\text {th }} \mathrm{ed}$, John Wiley and Sons, New York.

Edenburn MW and Burns JR 1981 Shading analysis of a photovoltaic cell string illuminated by a parabolic through concentrator. In: Proceedings of the $15^{\text {th }}$
IEEE Photovoltaic Specialists Conference, 12-15 May, Kissimmee, Florida, pp.63-68.

Gordon JM 1986 Non-imaging solar energy concentrators (CPCs) with fully illuminated flat receivers: a viable alternative to flatplate collectors. J. Sol. Energy Eng. 108(3): 252-256.

Green MA 2006 Third Generation Photovoltaics: Advanced Solar Energy Conversion. Springer, Berlin.

IEA (International Energy Agency) 2017 Energy access outlook: from poverty to prosperity. World energy outlook special report. Paris, France.

James LW and Williams JK 1978 Fresnel optics for solar concentration on photovoltaic cells. In: proceedings of the $13^{\text {th }}$ IEEE photovoltaic specialists conferences, Washington DC, June 5-8; pp. 673-679.

O'Gallagher J and Winston R 1983 Development of compound parabolic concentrators for solar energy. Int. J. Ambient Energy 4(4): 171-186.

O'Gallagher JJ 2008 Non-imaging Optics in Solar Energy. Synthesis Lectures on Energy and the Environment: Technology, Science, and Society \#2. Morgan and Claypool, Illinois.

Paul DI 2019a Application of compound parabolic concentrators to solar photovoltaic conversion: A comprehensive review. Int. J. Energy Res. 43(9): 40034050.

Paul DI 2019b Review of mathematical equations for the design of compound parabolic concentrating solar energy collectors. Invertis J. Renew. Energy 9(3): 91-134.

Paul DI 2019c Optical performance analysis and design optimisation of multisectioned compound parabolic concentrators for photovoltaics application. Int. J. Energy Res. 43: 358-378.

Paul DI, Smyth M, Zacharopoulos A and Mondol J 2013 The design, fabrication and indoor experimental characterisation of an 
isolated cell photovoltaic module. Sol. Energy 88: 1-12.

Pfeiffer H and Bihler M 1982 The effects of non-uniform illumination of solar cells with concentrated light. Sol. Cells 5: 293-299.

Rabl A 1976a Comparison of solar concentrators. Sol. Energy 18(2): 93-111.

Rabl A 1976b Optical and thermal properties of compound parabolic concentrators. Sol. Energy 18(6): 497-511.

Sangrador J and Sala G 1979 Static concentrators for two-sided photovoltaic solar cells. Sol Energy 23: 53-60.

Solar radiation Data (SoDa) 2019 Copernicus Atmosphere Monitoring Service (CAMS) radiation service. Retrieved from http://www.soda-pro.com/soda-products [accessed on 10.12.2019].

Sørensen B, Breeze P, Suppes GJ, Bassam N, Silveira S, Yang S, da Rosa AV, Gupta HK, Roy S, Doble M, Broussely M, Maegaard P, Barbir F, Pistoia G, Kalogirou S and Storvick T 2009 Renewable Energy Focus e-Mega Handbook $1^{\text {st }}$ ed. (pp. 335-399), Elsevier, Amsterdam.

Su Y, Riffat SB and Pei G 2012 Comparative study on annual solar energy collection of a novel lens-walled compound parabolic concentrator (lens-walled CPC). Sustain. Cities Soc. 4: 35-40.

UN (United Nation) 2014 Energy: a brief discussion on goals, targets and indicators. UN-Energy, Background note. New York.

Welford WT and Winston R 1978 The Optics of Non-imaging Concentrators. Academic Press, New York.

Winston R 1974 Principles of solar collectors of a novel design. Sol. Energy 16(2): 8995.

Winston R 1976 Photovoltaic applications of compound parabolic concentrator (CPC). Final report contract number W-31-109-ENG-38 (pp. 279-296) submitted to Argonne National laboratory, Argonne, Illinois.

Yu Y, Liu N and Tang R 2014 Optical performance of CPCs for concentrating solar radiation on flat receivers with a restricted incidence angle. Renew. Energy 62: 679-688.

Zacharopoulos A 2001 Optical design modelling and experimental characterisation of line-axis concentrators for solar photovoltaic and thermal applications. $\mathrm{PhD}$ thesis, University of Ulster. 